Dedicated to Professor Emeritus Ioan A. Rus on the occasion of his $80^{\text {th }}$ anniversary

\title{
Examples of cyclical operators
}

\section{ANDREI HORVAT-MARC and MiHAELA PETRIC}

\section{ABSTRACT.}

In this paper, we give examples of cyclic operators defined on various types of sets, in order to illustrate some results in the extremely rich literature following the seminal paper [Kirk, W. A., Srinivasan, P. S. and Veeramani, P., Fixed points for mappings satisfying cyclical contractive conditions, Fixed Point Theory, 4 (2003), No. 1, 79 - 89]. All examples which are presented enrich the list of cyclic operators and give a subject to future studies of this type of operators.

\section{REFERENCES}

[1] Bourdon, P. S. and Shapiro, J. H., Cyclic Phenomena for Composition Operators, Mem. Amer. Math. Soc. 125 (1997), no. 596, x+105 pp.

[2] Gao, J., Modulus of convexity in Banach spaces, Appl. Math. Lett., 16 (2003), No. 3, 273-278

[3] Eldred, A. and Veeramani, P., Existence and convergence of best proximity points, J. Math. Anal. Appl., 323 (2006), No. 2, 1001-1006

[4] Halmos, P. R. and Halmos, P. P. R., A Hilbert Space Problem Book, Graduate Texts in Mathematics, Springer, 1982

[5] Horvat-Marc, A. and Berinde, M., Another general fixed point principle, Carpathian J. Math., 20 (2004), No. 1, 45-49

[6] Karapınar, E., Romaguera, S. and Taş, K., Fixed points for cyclic orbital generalized contractions on complete metric spaces, Cent. Eur. J. Math., 11 (2013), No. 3, 552 - 560

[7] Karpagam, S. and Zlatanov, B., A note on best proximity points for P summing cyclic orbital Meir-Keeler contractions, Int. J. Pure Appl. Math., 107 (2016), No. 1, 225-243

[8] Kirk, W. A., Srinivasan, P. S. and Veeramani, P., Fixed points for mappings satisfying cyclical contractive conditions, Fixed Point Theory, 4 (2003), No. 1, 79-89

[9] Păcurar, M., and Rus, I. A., Fixed point theory for cyclic $\varphi$-contractions, Nonlinear Anal. 72 (2010), no. 3-4, 1181-1187.

[10] Păcurar, M., Fixed point theory for cyclic Berinde operators, Fixed Point Theory, 12 (2011), No. 2, 419-428

[11] Petric, M. A., Fixed points and best proximity points theorems for cyclical contractive operators, Ph.D. Thesis, North University of Baia Mare, 2011

[12] Petric, M. A., Zlatanov, B., Best proximity points and fixed points for p-summing maps, Fixed Point Theory Appl., 86 (2012), 145-154

[13] Petric, M. A., Best proximity point theorems for weak cyclic Kannan contractions, Filomat, 25 (2011), No. 1, 145-154

[14] Petric, M. A., Some results concerning cyclical contractive mappings, Gen. Math., 18 (2010), No. 4, 213-226

[15] Petric, M. A., Some remarks concerning Ciric-Reich-Rus operators, Creat. Math. Inform., 18 (2010), No. 2, 188-193

[16] Petric, M. A. and Zlatanov, B., Fixed point theorems of Kannan type for cyclical contractive conditions, University Press" Paisii Hilendarski", Plovdiv, 2010, 187-194

[17] Petruşel, G., Cyclic representation and periodic points, Studia Univ. Babeş-Bolyai Math., 50 (2005), No. 3, 107-112

Received: 21.06.2016; In revised form: 14.07.2016; Accepted: 22.07.2016

2010 Mathematics Subject Classification. 47H10, 54H25.

Key words and phrases. Fixed point theorems, cyclic operator, best proximity points.

Corresponding author: Andrei Horvat-Marc; hmandrei@cunbm.utcluj.ro 
[18] Rus, I. A., Mureşan, S. and Miklos, E., Maximal fixed point structures, Studia Univ. Babeş-Bolyai Math., 48 (2003), No. 3, 141-145

[19] Rus, I. A., Fixed point structures, Mathematica (Cluj), 28 (51) (1986), 59-64

[20] Wu, P. Y., Sums and products of cyclic operators, Proc. Amer. Math. Soc., 122 (1994), No. 4, 1053-1063

[21] Zlatanov, B., Error estimates for approximating best proximity points for cyclic contractive maps, Carpathian J. Math., 32 (2016), No. 2, 265-270

Department OF MATHEMATIC AND COMPUTER SCIENCES

NORTH University CENTER AT BAiA MARE

TECHNICAL UNIVERSITY OF CLUJ-NAPOCA

Victoriei 76, 430122 BAiA MARE, ROMANIA

E-mail address: hmandrei@cunbm.utcluj.ro 